\title{
MATURAÇÃO SEXUAL E APTIDÃO FÍSICA DE ESCOLARES DE ILHABELA
}

\author{
SEXUAL MATURITY AND PHYSICAL FITNESS OF SCHOOLCHILDREN OF ILHABELA
}

\section{Gerson Luis de Morais Ferrari ${ }^{a^{*}}$, Rodrigo Mateus Farias ${ }^{b^{* *}}$, Tatiane Kosimenko Ferrari ${ }^{* * *}$, Victor Keihan Rodrigues Matsudo ${ }^{\mathrm{d}^{* *}}$}

agersonferrari08@yahoo.com.br, bmateusfarias@gmail.com, ctatianekferrari@yahoo.com.br, dmatsudo@celafiscs.org.br *Universidade Federal de São Paulo - São Paulo (SP), Brasil

**Centro de Estudos do Laboratório de Aptidão Física de São Caetano do Sul - São Caetano do Sul (RS), Brasil

\section{RESUMO}

Introduçáo: $\mathrm{O}$ desenvolvimento adequado das capacidades físicas está diretamente relacionado com uma melhor qualidade de vida em todas as fases da vida. Objetivo: Verificar a associaçáo entre a maturaçáo sexual e a aptidão física de escolares de 7 a 18 anos do município de Ilhabela. Métodos: o estudo faz parte do Projeto Misto-Longitudinal de Crescimento, Desenvolvimento e Aptidão Física de Ilhabela. Participaram do estudo 1.156 escolares, de ambos os sexos, entre 7 e 18 anos de idade. As variáveis analisadas foram: massa corporal, estatura, adiposidade, força de membros inferiores e superiores, abdominais, flexibilidade, agilidade, velocidade, e aptidão cardiorrespiratória. A maturação sexual foi realizada de acordo com o método de autoavaliação dos pelos púbicos (P1 a P5). Foi feito a análise de regressão linear e one-way ANOVA, seguido do post hoc de Bonferroni. O nível de significância adotado foi $\mathrm{p}<0,05$. Resultados: No sexo masculino, observou-se que o percentual de gordura corporal foi $11,4 \%$ maior em P1 ( $p=0,04)$, e 10,2\% maior em P3 ( $\mathrm{p}=0,01)$, comparado ao $\mathrm{P} 5$. As diferenças entre os estágios maturacionais ocorreram na flexibilidade $(p=0,03)$, nos testes de abdominais $(p=0,04)$ e na flexáo de braços $(p<0,01)$, entre P2 e P5. No sexo feminino, valores médios de aptidáo cardiorrespiratória foram inferiores $(\mathrm{p}<0,01)$ para aquelas nos estágios finais de maturação. Conclusão: houve associação entre a maturação sexual avaliada a partir do desenvolvimento dos pelos púbicos (P1 a P5) e as variáveis de aptidão física de escolares de ambos os sexos no município de Ilhabela, antes e após o ajuste para massa corporal, estatura, IMC e adiposidade.

Palavras-chave: Estudantes; aptidão física; crescimento; desenvolvimento.

\section{ABSTRACT}

Introduction: the development of physical skills is directly related to a better quality of life at all stages of life. Objective: verify the association between sexual maturation and physical fitness of schoolchildren aged 7 to 18 years in the city of Ilhabela. Methods: The study is part of the Mixed Longitudinal Project on Growth, Development and Physical Fitness of Ilhabela. The study included 1,156 students of both sexes, aged 7 to 18 years old. The variables analyzed were: body weight, height, body fat, strength of upper and lower limbs, abdominal, flexibility, agility, speed, and cardiorespiratory fitness. Sexual maturation was analyzed according to the self-assessment method of pubic hair (P1 to P5). The linear regression analysis was performed and one-way ANOVA followed by post hoc Bonferroni. The level of significance was $\mathrm{p}<0.05$. Results: In boys, we found that the percentage of body fat was $11.4 \%$ higher in P1 ( $=0.04)$ and $10.2 \%$ higher in $\mathrm{P} 3(\mathrm{p}=0.01)$, compared to $\mathrm{P} 5$. The differences between maturational stages were flexibility ( $\mathrm{p}=0.03)$ in the abdominal $(\mathrm{p}=0.04)$ and the test push-up $(\mathrm{p}<0.01)$ between P2 and P5. In girls, mean values of cardiorespiratory fitness were lower $(p<0.01)$ for those in the final stages of maturation. Conclusion: there was association between sexual maturation evaluated from the development of pubic hair (P1 to P5) and physical fitness variables of students from both sexes in the city of Ilhabela, before and after adjusting for body weight, height, BMI and adiposity.

Keywords: Students; physical fitness; growth; development. 


\section{Introdução}

A capacidade do ser humano de realizar tarefas diárias com vigor e demonstrar traços e características que estáo associados ao baixo risco de desenvolvimento prematuro de doenças hipocinéticas depende do nível de aptidão física ${ }^{1}$. Seus componentes englobam diferentes dimensóes, podendo contemplar aspectos relacionados à saúde ou às habilidades esportivas ${ }^{2}$. Os componentes da aptidão física relacionados à saúde incluem variáveis como a aptidão cardiorrespiratória, força, flexibilidade e composição corporal. Já os componentes relacionados às habilidades esportivas incluem variáveis como a agilidade, equilíbrio, coordenação motora, potência e velocidade ${ }^{2}$.

Entre os componentes da aptidão física, a cardiorrespiratória é considerada um dos principais marcadores de condiçóes de saúde, em todas as idades ${ }^{3-5}$. Níveis adequados de aptidão cardiorrespiratória em crianças e adolescentes associam-se inversamente a fatores de risco cardiovasculares e metabólicos ${ }^{6-8}$. Além disso, todos os outros componentes também estáo fortemente associados à qualidade de vida dos indivíduos em todas as fases da vida ${ }^{9-11}$.

Esses componentes podem sofrer influência da ação dos hormônios sexuais decorrentes das modificaçôes que ocorrem com a maturação sexual, como o ganho acentuado de adiposidade no sexo feminino e o de massa muscular no sexo masculino ${ }^{12-15}$. A maturação sexual é caracterizada por alteraçóes físicas e biológicas, que ocorrem durante a puberdade. Este período é marcado pelo desenvolvimento de características sexuais secundárias, como o desenvolvimento de genitais no sexo masculino e dos seios no sexo feminino, assim como o surgimento de pelos púbicos em ambos os sexos ${ }^{16}$.

Alguns estudos já demonstraram que, dentro de um determinado grupo de idade, alguns indivíduos podem apresentar vantagens ou desvantagens em testes de aptidão cardiorrespiratória de acordo com a maturação sexual ${ }^{17-20}$. Porém, ainda sáo escassos os estudos em grande escala que descrevem como os diversos componentes da aptidão física diferem de acordo com a maturação sexual.

Sendo assim, o objetivo do presente estudo foi verificar a associação entre a maturação sexual e a aptidão física de escolares entre 7 e 18 anos do município de Ilhabela, litoral norte do estado de São Paulo.

\section{Métodos}

O presente estudo faz parte do Projeto MistoLongitudinal de Crescimento, Desenvolvimento e Aptidão Física de Ilhabela, desenvolvido pelo Centro de Estudos do Laboratório de Aptidão Física de São Caetano do Sul (CELAFISCS) desde 1978, com avaliações semestrais, nos meses de abril e outubro. Todas as avaliações são realizadas por profissionais qualificados da área da saúde, com o objetivo de mensurar variáveis antropométricas, neuromotoras e metabólicas, nível de atividade física e nutricional de crianças e adolescentes a partir dos sete anos de idade, do município de Ilhabela (São Paulo - Brasil), mediante uma bateria de testes e medidas padronizadas pelo nosso centro ${ }^{21,22}$. Esse projeto é capaz de fornecer dados úteis para a elaboração de estratégias preventivas contra doenças crônicas não transmissíveis, consideradas hoje um grande problema, que ameaça a saúde pública no Brasil e em outros países em desenvolvimento ${ }^{23}$.

\section{Amostra}

Para compor a amostra deste estudo, foi analisado um banco de dados composto de mais de 16.000 escolares, de ambos os sexos, sendo que 1.156 escolares (608 meninos e 547 meninas) atenderam aos seguintes critérios de inclusão: (a) apresentar avaliação física completa entre 2008 e 2011; (b) possuir entre 7 e 18 anos de idade; (c) estar regularmente matriculado na rede de ensino de Ilhabela; (d) não possuir limitaçóes clínicas e funcionais para realização dos testes de esforço; (e) apresentarem o Termo de Consentimento Livre e Esclarecido, assinado pelos pais ou responsáveis, conforme a Resoluçáo 196/96 do Conselho Nacional de Saúde. O projeto foi aprovado pelo Comitê de Ética da Universidade Federal de São Paulo sob o protocolo 0056/10.

\section{Métodos}

As variáveis analisadas foram: massa corporal, estatura, adiposidade, força de membros inferiores e superiores, abdominais, flexibilidade, agilidade, velocidade, e aptidão cardiorespiratória, de acordo com a padronização CELAFISCS ${ }^{21}$. A caracterização da amostra participante do estudo é apresentada na Tabela 1.

A medida da massa corporal $(\mathrm{kg})$ foi obtida mediante a utilização de uma balança (Filizola modelo Personal Life) digital, com precisão de 100 gramas, com o indivíduo trajando roupas leves. Em seguida, colocamos os participantes no centro da plataforma, eretos, com seu olhar em um ponto fixo, à sua frente. A estatura foi obtida por um estadiômetro, com base fixa e cursor móvel, em posição ortostática, pés descalços e unidos, encostando a superfície posterior do calcanhar, cintura pélvica, cintura escapular e região 
occipital no instrumento. A medida foi feita com o indivíduo em apneia inspiratória, minimizando possíveis variaçóes sobre a variável antropométrica. A cabeça foi posicionada de acordo com o plano de Frankfurt, paralela ao solo, com seu cursor no ângulo de 90 graus em relação à escala, e calculada pela média de três mensuraçóes. Para o cálculo do índice de massa corporal (IMC) foi utilizada a fórmula de peso corporal, dividida pela altura ao quadrado.
A adiposidade $(\mathrm{mm})$ foi obtida através da determinação de sete dobras cutâneas (bíceps, tríceps, subescapular, suprailíaca, axilar média, abdômen e panturrilha), realizadas pelo mesmo profissional em todas as avaliaçóes do projeto. As medidas foram feitas no hemicorpo direito realizando três medidas sucessivas no mesmo local, adotando como valor final a média dessas medidas ${ }^{21}$. O modelo de compasso utilizado foi o Harpenden ${ }^{\grave{o}}$, calibrado previamente.

Tabela 1: Caracterização geral da amostra do município de Ilhabela, Brasil.

\begin{tabular}{|c|c|c|c|c|c|c|c|c|c|}
\hline \multirow{2}{*}{ Variáveis } & \multicolumn{4}{|c|}{ Sexo Masculino $(n=608)$} & \multicolumn{4}{|c|}{ Sexo Feminino $(n=547)$} & \multirow{2}{*}{ p valor } \\
\hline & $\mathbf{n}$ & $\overline{\mathrm{X}} \pm \mathrm{DP}$ & Mín & Máx & $\mathbf{n}$ & $\overline{\mathrm{X}}_{ \pm \mathrm{DP}}$ & Mín & Máx & \\
\hline Idade (anos) & 608 & $11,8 \pm 2,17$ & 7 & 18 & 547 & $11,70 \pm 11,70$ & 7 & 18 & 0,339 \\
\hline Massa Corporal (kg) & 600 & $41,90 \pm 12,55$ & 19,4 & 94,6 & 547 & $43,38 \pm 11,68$ & 19,9 & 85,5 & 0,040 \\
\hline Estatura $(\mathrm{cm})$ & 588 & $149,62 \pm 13,60$ & 114,1 & 188,2 & 545 & $150,19 \pm 10,87$ & 119,1 & 171,2 & 0,432 \\
\hline $\operatorname{IMC}\left(\mathrm{kg} / \mathrm{m}^{2}\right)$ & 608 & $18,32 \pm 2,92$ & 10,98 & 30,23 & 545 & $18,93 \pm 3,41$ & 11,57 & 39,83 & $<0,001$ \\
\hline $\bar{X} 7 \mathrm{DC}(\mathrm{mm})$ & 604 & $9,33 \pm 4,95$ & 4,1 & 31,5 & 537 & $13,26 \pm 5,91$ & 4,2 & 52,8 & $<0,001$ \\
\hline Força MMII (cm) & 600 & $28,97 \pm 9,49$ & 14 & 93 & 537 & $25,78 \pm 7,15$ & 9 & 85 & $<0,001$ \\
\hline Força MMSS (kg) & 604 & $23,34 \pm 9,80$ & 9 & 58 & 543 & $21,35 \pm 6,40$ & 9 & 46 & $<0,001$ \\
\hline Abdominal (rep) & 595 & $33,96 \pm 9,66$ & 4 & 70 & 515 & $27,13 \pm 8,87$ & 1 & 71 & $<0,001$ \\
\hline Flexibilidade $(\mathrm{cm})$ & 601 & $24,27 \pm 7,09$ & 4 & 49 & 539 & $27,99 \pm 7,41$ & 1 & 45 & $<0,001$ \\
\hline Agilidade (seg) & 603 & $12,31 \pm 1,25$ & 9,05 & 16,82 & 537 & $13,34 \pm 1,34$ & 9,93 & 21,22 & $<0,001$ \\
\hline Velocidade (seg) & 595 & $9,57 \pm 1,45$ & 6,50 & 28,75 & 527 & $10,46 \pm 1,23$ & 6,23 & 19,97 & $<0,001$ \\
\hline $\mathrm{VO}_{2 \text { máx }}\left(\mathrm{ml} \cdot \mathrm{kg}^{-1} \cdot \mathrm{min}^{-1}\right)$ & 608 & $36,28 \pm 8,75$ & 13,51 & 61,75 & 547 & $30,91 \pm 8,07$ & 13,54 & 61,75 & $<0,001$ \\
\hline
\end{tabular}

p <0,05; $\bar{X}$ : média; DP: desvio padrão; Mín: mínimo; Máx: máximo.

A força de membros inferiores foi obtida pelo teste de impulsão vertical, sem auxílio dos membros superiores (IVS), medido em centímetros. Os testes foram realizados três vezes, considerando para o registro o melhor resultado obtido.

A força de membros superiores $(\mathrm{kg})$ foi medida por um dinamômetro de preensão manual ajustável (Takei TK005, Tóquio, Japão). O participante foi orientado a apertar o dinamômetro vigorosamente com a mão direita, segurando o aparelho com certa distância do corpo, com o braço estendido. $\mathrm{O}$ teste foi executado duas vezes, com intervalo de 2 minutos, registrando-se o melhor valor de cada um.

A resistência dinâmica abdominal de flexão dos músculos do quadril foi medida em posição supina, com quadris e joelhos flexionados, e os pés fixados no chão. Foi registrada a quantidade de repetiçóes em um minuto.

A medida de flexibilidade $(\mathrm{cm})$ foi realizada pelo teste de sentar e alcançar, utilizando o banco de Wells. A agilidade (segundos) foi estimada através do teste de shuttle run (segundos). Ambos os testes foram realizados duas vezes, considerando o melhor resultado para a análise. A avaliação de velocidade (segundos) foi mensurada através do teste de corrida de 50 metros, realizado uma única vez. É importante ressaltar que, antes de cada sessão de teste, foi dada uma breve explicação a cada indivíduo sobre sua execução.

Para avaliar a aptidão cardiorrespiratória, foi estimada a potência aeróbica $\left(\mathrm{VO}_{2 \max }\right)$, mediante um protocolo progressivo submáximo em bicicleta ergométrica mecânica (Monark modelo Ergomedic 828E), com duração de 8 minutos e carga de aquecimento ( 4 minutos), e outra carga de trabalho calculada a partir do peso corporal (4 minutos). Os valores de pressão arterial, frequência cardíaca e percepção subjetiva de esforço foram medidos no repouso e a cada minuto do teste. Os escolares foram orientados a pedalar a $50 \mathrm{RPM}$. O VO $\mathrm{V}_{2 \max }$ é apresentado em valores relativos à massa corporal $\left(\mathrm{ml} . \mathrm{kg}^{-1} \cdot \mathrm{min}^{-1}\right)$ e, para o seu cálculo, utilizou-se o nomograma de Åstrand ${ }^{24}$, considerando a frequência cardíaca do último minuto da carga de esforço.

Para a determinação da maturação sexual, foi utilizado o critério estabelecido por Tanner ${ }^{25}$ mediante a técnica de autoavaliação das características sexuais secundárias de acordo com o sexo ${ }^{26}$.

Como critério de qualidade interna de todas as medidas e testes, foram calculadas a objetividade e 
reprodutibilidade das medidas obtidas em uma subamostra de 30 escolares, selecionados aleatoriamente em cada avaliaçáo. Foram encontrados os seguintes valores de objetividade e reprodutibilidade: peso corporal $(0,96$ e 0,99$)$; estatura $(0,97$ e 0,99$)$; dobras cutâneas $(0,92$ e $0,99)$; força de membros inferiores $(0,62$ e 0,92$)$; força de membros superiores $(0,51$ e 0,97$)$; abdominais $(0,49$ e 0,72$)$; flexibilidade $(0,61$ e 0,91$)$; agilidade $(0,58$ e $0,89)$; velocidade $(0,58$ e 092$)$; e potência aeróbica $(0,58$ e 0,88 ).

\section{Análise estatística}

Foi utilizada a estatística descritiva, com média, desvio padrão, frequência e porcentagem. Para verificar a distribuição dos dados, foi feito o teste de Kolmogorov$S_{\text {Smirnov }}^{27}$. Na comparação dos diferentes períodos de avaliação com as variáveis dependentes, foi aplicada a Análise de Variância com um fator, seguido pelo método de comparaçốes múltiplas de Bonferroni ${ }^{27}$.

A análise de regressão linear foi calculada para verificar a magnitude da influência da maturação sexual sobre as variáveis de aptidão física, ajustadas para idade e variáveis antropométricas (massa corporal, estatura, IMC e adiposidade). Os cálculos foram realizados pelo software Statistical Package for the Social Sciences (SPSS) versão 21.0, e o nível de significância adotado foi de $\mathrm{p}<0,05^{27}$.

\section{Resultados}

A Tabela 1 apresenta as características dos escolares (7 a 18 anos) de acordo com o sexo. Os meninos apresentaram valores maiores e estatisticamente significantes em força de membros inferiores e superiores, força abdominal e aptidão cardiorrespiratória, quando comparado ao sexo feminino ( $\mathrm{p}<0,001)$. Já as meninas apresentaram valores maiores do que os meninos em massa corporal, IMC, adiposidade corporal e flexibilidade. Para as variáveis de agilidade e velocidade, os meninos obtiveram melhores valores, se comparados às meninas.

A Tabela 2 apresenta a distribuição dos escolares de acordo com os diferentes estágios maturacionais dos pelos pubianos. O sexo masculino apresentou maiores proporçôes nos estágios P3, seguido pelo P2. Já nas meninas, as maiores proporçóes foram observadas no estágio P4, seguido pelo P2. Tanto o sexo masculino quanto o feminino tiveram maiores proporçóes no período púbere.

Tabela 2: Distribuição da amostra de acordo com o estágio de maturação sexual, Ilhabela, Brasil.

\begin{tabular}{|c|c|c|c|c|}
\hline \multirow{2}{*}{ Estágios } & \multicolumn{2}{|c|}{ Sexo Masculino } & \multicolumn{2}{|c|}{ Sexo Feminino } \\
\hline & $f$ & $\%$ & $f$ & $\%$ \\
\hline P1 & 74 & 12,5 & 101 & 20,5 \\
\hline P2 & 134 & 22,6 & 103 & 20,9 \\
\hline P3 & 173 & 29,1 & 61 & 12,4 \\
\hline P4 & 127 & 21,4 & 162 & 32,9 \\
\hline P5 & 86 & 14,5 & 66 & 13,4 \\
\hline Total & 594 & 100,0 & 493 & 100,0 \\
\hline
\end{tabular}

No sexo masculino (Figura 1), os valores médios de massa corporal, estatura, força de membros inferiores e flexibilidade foram significativamente inferiores $(\mathrm{p}<0,001)$ nos estágios pré-púberes (P1) e púberes (P2 a $\mathrm{P} 4)$, quando comparados ao estágio pós-púbere (P5).

Em relação ao IMC e à força de membros superiores, os valores médios foram significativamente inferiores nos estágios pré-púberes (P1) e nos estágios P2 e P3 dos púberes, quando comparados ao estágio pós-púbere (P5). Quanto ao desempenho nos testes de abdominais, os escolares pertencentes ao estágio pós-púbere (P5) tiveram melhor desempenho do que aqueles pertencentes aos estágios P1, P2 e P3 ( $<<0,001)$.
A agilidade melhorou com o avanço dos estágios dos pelos púbicos, sendo estatisticamente diferentes nos estágios púberes (P2, P3 e P4) quando comparados ao estágio pós-púbere (P5) $(\mathrm{p}<0,001)$. A velocidade, também melhorou com o avanço dos estágios, sendo estatisticamente diferente nos pré-púberes (P1) e púberes (P2, P3 E P4) quando comparados aos pós-púberes (P5) $(\mathrm{p}<0,001)$.

Em relação à aptidão cardiorrespiratória, foi observada uma diferença estatística somente entre os estágios de maturação sexual pré-púbere (P1) e pós-púbere (P5) $(\mathrm{p}<0,001)$. A adiposidade náo apresentou diferença estatística entre os estágios de maturação sexual dos pelos púbicos. 
Figura 1: Valores de média das variáveis da aptidão física no sexo masculino de acordo com os estágios de maturação sexual dos escolares de Ilhabela, Brasil.
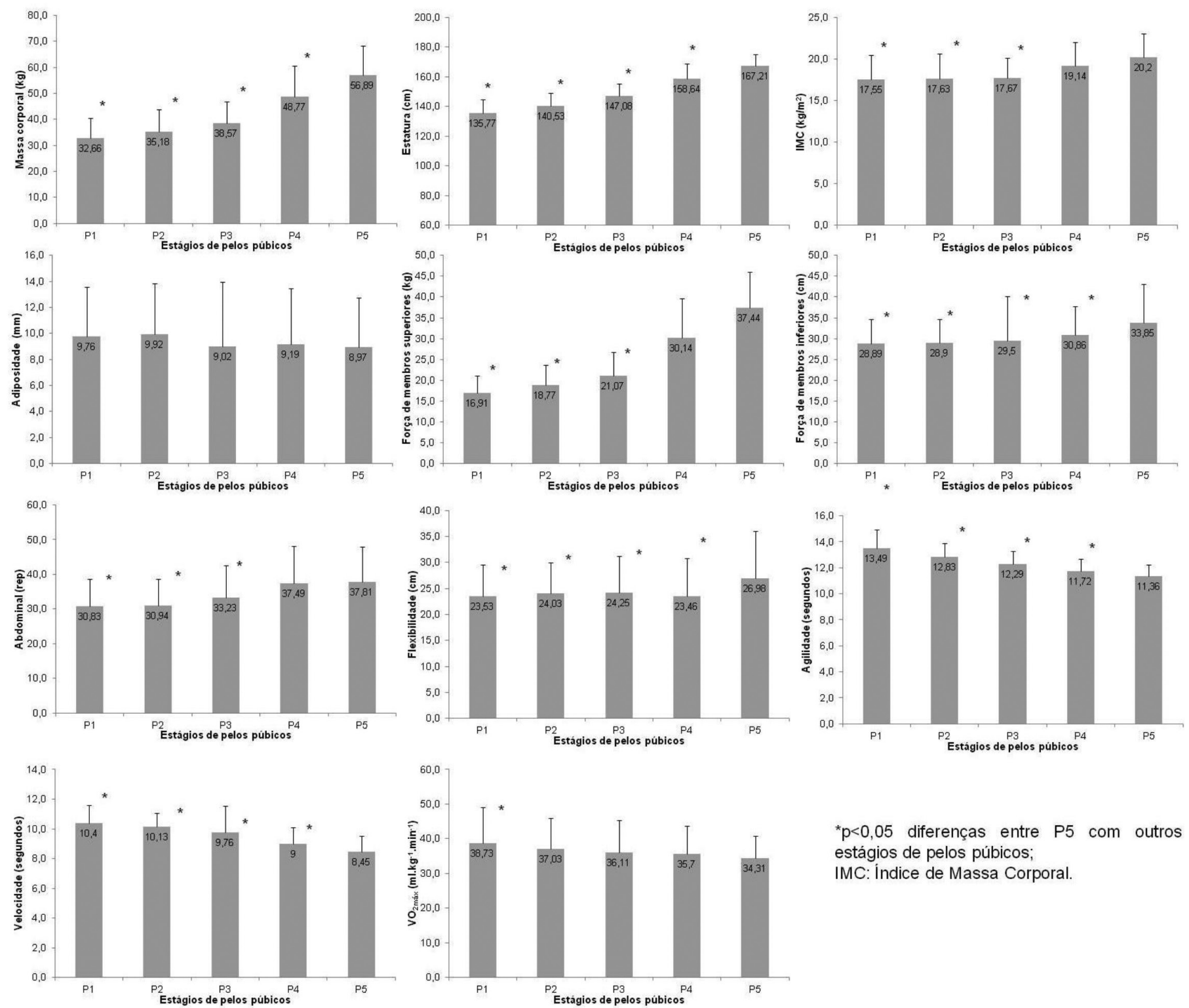

${ }^{*} \mathrm{p}<0,05$ diferenças entre P5 com outros estágios de pelos púbicos; IMC: Índice de Massa Corporal. 
Figura 2: Valores de média das variáveis da aptidão física no sexo feminino de acordo com os estágios de maturação sexual dos escolares de Ilhabela, Brasil.
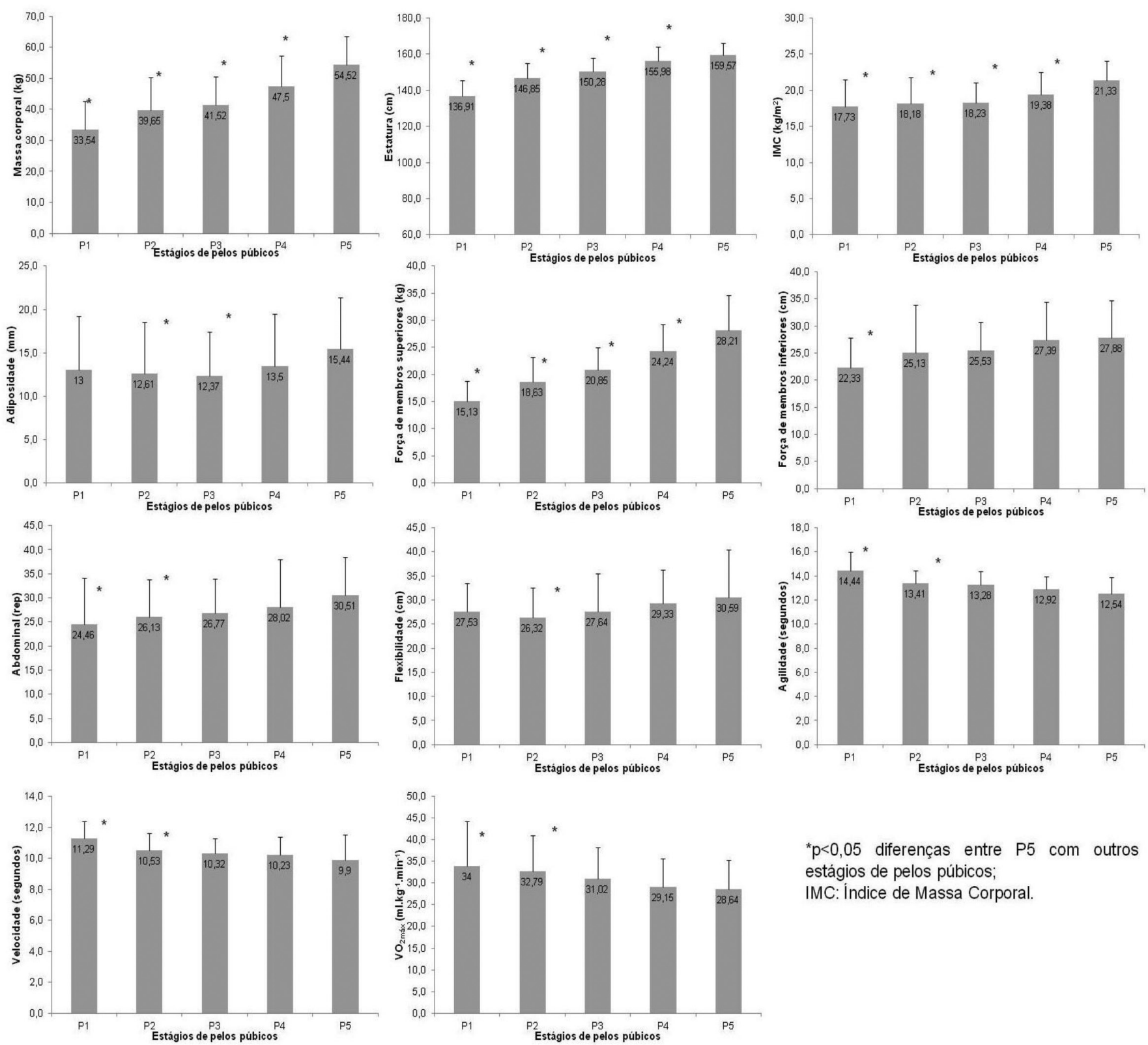

${ }^{*} p<0,05$ diferenças entre P5 com outros estágios de pelos púbicos; IMC: Índice de Massa Corporal. 
Tabela 3: Análise multivariada da associação entre os estágios de pelos púbicos com as variáveis de aptidão física de escolares de Ilhabela. Modelo de regressão linear: $\beta$ e intervalos de confiança (IC95\%).

\begin{tabular}{|c|c|c|c|c|c|}
\hline & $\beta$ & $95 \% \mathrm{CI}$ & SE & $p$ & $\mathbf{R}^{2}$ \\
\hline \multicolumn{6}{|c|}{ Meninos } \\
\hline Força MMII & $-0,425$ & $-1,400,0,551$ & 0,497 & 0,393 & 0,13 \\
\hline Força MMSS & 0,520 & $0,074,0,966$ & 0,227 & 0,022 & 0,80 \\
\hline Abdominal & $-0,015$ & $-0,914,0,883$ & 0,458 & 0,973 & 0,19 \\
\hline Flexibilidade & 0,97 & $0,257,1,683$ & 0,363 & 0,008 & 0,06 \\
\hline Agilidade & $-0,122$ & $-0,220,-0,024$ & 0,050 & 0,015 & 0,42 \\
\hline Velocidade & $-0,095$ & $-0,221,0,031$ & 0,064 & 0,138 & 0,33 \\
\hline 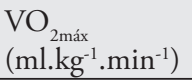 & 0,757 & $-0,048,1,563$ & 0,410 & 0,065 & 0,21 \\
\hline \multicolumn{6}{|c|}{ Meninas } \\
\hline Força MMII & 0,669 & $0,011,1,327$ & 0,335 & 0,046 & 0,15 \\
\hline \multicolumn{6}{|c|}{ Meninas } \\
\hline Força MMSS & 0,559 & $0,207,0,910$ & 0,179 & 0,002 & 0,69 \\
\hline Abdominal & 1,350 & $0,501,2,199$ & 0,432 & 0,002 & 0,12 \\
\hline Flexibilidade & 0,834 & $0,127,1,542$ & 0,360 & 0,021 & 0,04 \\
\hline Agilidade & $-0,127$ & $-0,237,-0,018$ & 0,056 & 0,023 & 0,34 \\
\hline Velocidade & $-0,064$ & $-0,174,0,045$ & 0,056 & 0,250 & 0,25 \\
\hline 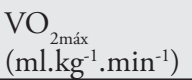 & 0,044 & $-0,634,0,722$ & 0,345 & 0,127 & 0,28 \\
\hline
\end{tabular}

No sexo feminino (Figura 2), os valores médios da massa corporal, estatura, IMC e força de membros superiores foram significativamente inferiores $(\mathrm{p}<0,001)$ nos estágios pré-púberes (P1) e púberes (P2 a P4) quando comparados ao estágio pós-púbere (P5).

Em relação à adiposidade, os valores médios foram significativamente inferiores nos estágios P2 e P3 dos púberes, quando comparados ao pós-púbere (P5). Quanto à força de membros inferiores, somente os pré-púberes (P1) apresentaram valores médios significativamente inferiores $(\mathrm{p}<0,001)$ do que os pós-púberes (P5). Já em relação à flexibilidade, somente o estágio P2 dos púberes apresentou valor médio significativamente inferior $(\mathrm{p}<0,001)$ do que os pós-púberes $(\mathrm{P} 5)$.

No que diz respeito ao desempenho dos testes abdominais, os escolares pertencentes ao estágio pós-púbere (P5) tiveram melhor desempenho do que aqueles pertencentes aos estágios P1e P2 ( $<0,001)$.

A agilidade, velocidade e aptidáo cardiorrespiratória melhoraram com o avanço dos estágios dos pelos púbicos, sendo estatisticamente diferentes dos estágios pré-púbere (P1) e do estágio $\mathrm{P} 2$ dos púberes, quando comparados aos pós-púberes (P5) ( $\mathrm{p}<0,001)$.

A Tabela 3 apresenta os resultados da regressão linear multivariada ajustada para idade e variáveis antropométricas (massa corporal, estatura, IMC e adiposidade). Podemos notar que os estágios de pelos púbicos (P1 a $\mathrm{P} 5, \mathrm{p}<0,001)$ do sexo masculino possuem associação significante com a força dos membros superiores, flexibilidade e agilidade.
Já no sexo feminino, podemos notar associação significante de todos os estágios de pelos púbicos ( $\mathrm{P} 1 \mathrm{a}$ P5) com a força dos membros inferiores, superiores e abdominal, junto da flexibilidade e agilidade.

\section{Discussão}

Os resultados do presente estudo demostraram associação entre a maturação sexual e as variáveis de aptidão física de escolares no município de Ilhabela. No sexo feminino, a maturação sexual avaliada a partir do desenvolvimento dos pelos púbicos (P1 a P5) foi associada com a massa corporal, estatura, IMC, adiposidade, força de membros superiores e inferiores, resistência abdominal, flexibilidade, agilidade, velocidade e aptidão cardiorrespiratória. Já no sexo masculino, a maturação sexual foi associada com todas as variáveis anteriormente citadas, exceto a adiposidade. Ao analisar a associaçáo entre a maturação sexual e as variáveis de aptidão física ajustadas para a massa corporal, estatura, IMC e adiposidade no sexo masculino, foi observada associação significante entre: força dos membros superiores e estágios P1 a P5; flexibilidade e estágio P4; agilidade e estágio P1 de desenvolvimento de pelos púbicos. Já no sexo feminino, foi observada associaçáo significante entre: força dos membros superiores e estágios P1 a P5 e; agilidade e estágio P1 no desenvolvimento de pelos púbicos.

Soares et al. ${ }^{20}$ verificaram a influência da maturação sexual na aptidão cardiorrespiratória de escolares com 
idade entre 9 e 14 anos da rede pública de Aracajú (SE). Os resultados revelaram uma associação entre a maturação sexual e a aptidão cardiorrespiratória no sexo feminino, ocasionando uma tendência decrescente e linear de acordo com a evolução dos estágios de desenvolvimento dos pelos púbicos. Esses resultados são semelhantes aos do nosso estudo, que também observou uma associação entre a aptidão cardiorrespiratória e a maturação sexual, além de uma tendência decrescente de acordo com a evolução dos estágios de desenvolvimento dos pelos púbicos. Porém, no presente estudo, esses resultados também foram observados no sexo masculino.

Essa associação também foi realizada por Minatto, Petroski e Silva ${ }^{28}$, em que foi analisada a aptidão física relacionada à saúde, de acordo com os estágios de maturação sexual em adolescentes brasileiros de $10 \mathrm{a}$ 17 anos de idade, residentes em uma cidade pequena, de colonização germânica. As análises demonstraram diferenças entre os estágios de maturação sexual no percentual de gordura corporal e na aptidão muscular no sexo masculino, e também na aptidáo cardiorrespiratória no sexo feminino. Apesar de não avaliar o percentual de gordura, os resultados desse estudo foram semelhantes aos encontrados com os escolares em Ilhabela. É importante ressaltar que esta amostra demonstrou associaçóes entre maturação sexual e aptidão cardiorrespiratória e muscular para ambos os sexos.

No estudo de Ortega et al. ${ }^{14}$ foram determinados os níveis de diferentes componentes de aptidão física, relacionadas com a idade cronológica e biológica de adolescentes espanhóis. Foram avaliados 2.859 indivíduos (1.357 homens e 1.502 mulheres), por meio de testes específicos. Os resultados revelaram que a força muscular foi maior nos adolescentes mais velhos do que nos mais novos. Por outro lado, a capacidade cardiorrespiratória foi maior nos mais novos, bem como na puberdade precoce em comparação à tardia. Um dado interessante encontrado no sexo masculino foi a maior aptidáo cardiorrespiratória nos mais novos, quando analisada a idade cronológica, porém no que diz respeito à maturação sexual não foi encontrada diferença significativa entre os grupos. Foi possível concluir nessas análises que a discrepância entre idade cronológica e idade biológica foi maior na aptidão cardiorrespiratória do que na força muscular. De acordo com resultados do estudo anterior, no qual demonstra-se perda de capacidade cardiorrespiratória com o passar do tempo, os dados de um estudo longitudinal, que avaliou adolescentes belgas entre 1969 e 2005, apresentou aumento de peso, estatura, IMC e dobras cutâneas, além da diminuição do desempenho em vários testes de aptidão física ${ }^{29}$.

Em outro seguimento longitudinal, que avaliou a influência da maturação sexual na aptidão física de escolares, Ferrari et al. ${ }^{30}$ analisaram escolares do sexo masculino, entre 10 e 13 anos de idade, que foram acompanhados durante quatro anos. Os autores verificaram que a maturação sexual explicou, principalmente, a adiposidade, mas também a força, velocidade e potência aeróbica absoluta $\left(1 . \mathrm{min}^{-1}\right)$. Enquanto isso, ao analisar transversalmente a associação entre a maturação sexual e as variáveis de aptidão física ajustadas para a massa corporal, estatura, IMC e adiposidade no sexo masculino, o presente estudo mostrou associação significante entre: força dos membros superiores e estágios P1 a P5; flexibilidade e estágio P4; agilidade e estágio P1 de desenvolvimento de pelos púbicos.

Parece evidente que a maturaçáo biológica influencia o desempenho de testes de aptidão física. Crianças e adolescentes podem apresentar vantagens ou desvantagens, sendo mais ou menos maduros do que seus colegas de mesma idade cronológica, como demonstrado em um estudo transversal, envolvendo 313 meninos e meninas, submetidos à avaliação antropométrica de maturação sexual, força e agilidade. Pode-se notar que a maturidade sexual tem influência sobre as medidas de aptidão física em meninos e meninas (meninos: $r=0,56-0,73$; meninas: $r=0,24-$ $0,46)^{31}$. Nesse mesmo sentido, um grupo de pesquisadores portugueses publicou, em 2002, um estudo que analisou as mudanças na composição corporal e na aptidão cardiorrespiratória, por meio dos estágios de maturação. Foram analisados os dados de meninos e meninas de 8 a 16 anos de idade, e foram constatadas diferenças significativas em todos os estágios de maturação no sexo feminino. Entre os meninos, apenas peso e altura diferiram significativamente pelo estágio de maturação sexual ${ }^{19}$.

Os resultados apresentados fortalecem o conceito de que a maturaçáo sexual explica diretamente as variáveis de aptidão física em algumas idades cronológicas. Entre estas variáveis, a aptidão cardiorrespiratória é considerada um dos principais componentes da aptidão física relacionada à saúde ${ }^{3-5}$. Nesse contexto, é importante que se estabeleça níveis adequados de aptidão cardiorrespiratória na adolescência, pois fatores de risco cardiovasculares e metabólicos estão diretamente relacionados a baixos níveis de aptidáo física em geral $^{6-8}$.

Entendemos que os resultados do presente estudo são úteis e de fundamental importância para o planejamento de políticas públicas, direcionadas à promoção da saúde de crianças e adolescentes, permitindo a realização de intervençóes de acordo com os estágios de maturação sexual, favorecendo significativamente a melhora da qualidade de vida da população. 


\section{Limitações}

Apesar de nosso estudo fazer parte de um projeto misto longitudinal realizado há mais de 30 anos, e possuir uma amostra considerável demais de 1000 indivíduos, os autores consideram que a investigação atual possui algumas limitaçóes: a) a amostra analisada não é considerada representativa para essa população; b) o valor médio da qualidade de algumas variáveis, entre as décadas, teve variação de moderada a alta; c) não foi realizada avaliação do nível de atividade física e do comportamento sedentário dos escolares e d) náo foram considerados os aspectos sociodemográficos, culturais e étnicos da populaçáo estudada.

\section{Conclusão}

A partir dos resultados apresentados no presente estudo, pode-se concluir que houve associação entre a maturação sexual avaliada a partir do desenvolvimento dos pelos púbicos ( $\mathrm{P} 1$ a $\mathrm{P} 5)$ e as variáveis de aptidão física de escolares no município de Ilhabela, antes e depois do ajuste para massa corporal, estatura, IMC e adiposidade.

Antes do ajuste, no sexo masculino, a maturação sexual foi associada com todas as variáveis estudadas, exceto a adiposidade. Já no sexo feminino, a maturaçáo sexual foi associada com a massa corporal, estatura, IMC, adiposidade, força de membros superiores e inferiores, resistência abdominal, flexibilidade, agilidade, velocidade e aptidão cardiorrespiratória. Após o ajuste, no sexo masculino, foi observada associação significante entre força dos membros superiores e estágios P1 a P5, flexibilidade e estágio P4, e agilidade e estágio P1 do desenvolvimento de pelos púbicos. Já no sexo feminino, foi observada associação significante entre força dos membros superiores e estágios P1 a P5, e agilidade e estágio P1 do desenvolvimento de pelos púbicos.

\section{Agradecimentos}

Fundação de Amparo à Pesquisa do Estado de São Paulo (FAPESP - 2010/20749-8); Coordenação de Aperfeiçoamento de Pessoal de Nível Superior (CAPES) e Conselho Nacional de Desenvolvimento Científico e Tecnológico (CNPq - 47807/2011-8). Participantes; pais e responsáveis; professores; coordenação da Escola Municipal José Antônio Verzegnassi e Escola Municipal Eva Esperança; e Prefeitura Municipal de Ilhabela.

\section{Referências}

1. Pate, R.R. The evolving definition of physical fitness. Quest Champaign. 1988;40(3):174-9.

2. Guedes DP, Guedes JERP. Atividade física, aptidáo física e saúde. Rev Bras Ativ Fis Saúde. 1995;1(1):18-35.

3. Myers J, Prakash M, Froelicher V, Do D, Partington S, Atwood JE. Exercise capacity and mortality among men referred for exercise testing. N Engl J Med. 2002;346:793-801.

4. Gulati M, Pandey DK, Arnsdorf MF, Laudesdale DS, Thisted RA, Wicklund RH, et al. Exercise capacity and the risk of death in women: the St James Women Take Heart Project. Circulation. 2003;108:1554-9.

5. Andersen LB, Harro M, Sardinha LB, Froberg K, Ekelund $\mathrm{U}$, Brage $\mathrm{S}$, et al. Physical activity and clustered cardiovascular risk in children: a cross-sectional study (The Europen Youth Heart Study). Lancet. 2006;368:299-304.

6. Twisk JWR, Kemper HCG, Van Mechelen W. The relationship between physical fitness and physical activity during adolescence and cardiovascular disease risk factors at adult age. The Amsterdam growth and health longitudinal study. Int J Sports Med. 2002;23:8-14.

7. Janz KF, Dawson JD, Mahoney LT. Increases in physical fitness during childhood improve cardiovascular health during adolescence: the Muscatine study. Int J Sports Med. 2002;23:15-21.

8. Lefevre J, Philippaerts R, Delvaux K, Thomis M, Claessens AL, Lysens $\mathrm{R}$, et al. Relation between cardiovascular risk factors at adult age, and physical activity during youth and adulthood: the Leuven Longitudinal Study on Lifestyle, Fitness and Health. Int J Sports Med. 2002;23:32-8.

9. Guedes DP, Guedes JERP, Guedes RP, Barbosa DS, Oliveira JA. Atividade física habitual e aptidão física relacionada à saúde em adolescentes. Rev Bras Ciên Mov. 2002;10(1):13-21.

10. Glaner MF. Aptidão física relacionada à saúde de adolescentes rurais e urbanos em relação a critérios de referência. Rev Bras Educ Fís Esp. 2005;19(1):13-24.

11. Canoy D. Distribution of body fat and risk of coronary heart disease in men and women. Curr Opin Cardiol. 2008;23(6):591-8.

12. Guedes DP, Neto JT, Silva AJ. Motor performance in a sample of Brazilian schoolchildren. Motricidade. 2011;7:25-38.

13. Pasquarelli BN, Silva VO, Bismarck-Nasr EM, Loch MR, Leão-Filho IB. Pubertal stage and overweight in school children from São José dos Campos, SP. Rev Bras Cineantropom Desempenho Hum. 2010;12:338-44.

14. Ortega FB, Ruiz JR, Castillo MJ, Moreno LA, Urzanqui A, González-Gross $M$ et al. Health-related physical fitness according to chronological and biological age in adolescents. The AVENA study. J Sports Med Phys Fitness. 2008;48:371-9.

15. Ortega FB, Ruiz JR, Mesa JL, Gutiérrez A, Sjöström M. Cardiovascular fitness in adolescents: the influence of sexual 
maturation status-the AVENA and EYHS studies. Am J Hum Biol. 2007;19:801-8.

16. Biro FM, Huang B, Daniels SR, Lucky AW. Pubarche as well as thelarche may be a marker for the onset of puberty. J Pediatr Adolesc Gynecol. 2008;21:323-8.

17. Beunen GP, Malina RM, Renson R, Simons J, Ostyn M, Lefevre J. Physical activity and growth, maturation and performance: a longitudinal study. Med Sci Sports Exer. 1992;24:576-85.

18. Bouchard C. Genetic aspects of human obesity. In: Bjorntorp P, Brodoff BN, editors. Obesity. New York: Lippincott; 1992. p. 343-51.

19. Mota J, Guerra S, Leandro C, Pinto A, Ribeiro JC, Duarte JA: Association of maturation, sex, and body fat in cardiorespiratory fitness. Am J Hum Biol. 2002; 14(6):707-12.

20. Soares NMM, Silva RJS, Melo EV, Oliveira ACC. Influência da maturaçáo sexual na aptidão cardiorrespiratória em escolares. Rev Bras Cineantropom Desempenho Hum. 2014;16(2):223-32.

21. Matsudo VKR. Testes em ciências do esporte. São Paulo: Gráficos Burti; 1998.

22. Ferrari GLM, Farias RM, Matsudo SMM, Matsudo VKR. Uma revisão de três décadas do projeto misto-longitudinal de crescimento, desenvolvimento e aptidão física de Ilhabela. Rev Bras Cien Saúde. 2013;11(36):57-67.

23. Ferrari GLM, Bracco MM, Matsudo VRM, Fisberg M. Cardiorespiratory fitness and nutritional status of schoolchildren: Evolution of 30 years. J Pediat. 2013;89:366-73.
24. Åstrand PO, Ryhming I. A nomogram for calcualtion of aerobic capacity (physical fitness) from pulse rate during submaximal work. J Appl Physiol. 1954;7:218-21.

25. Tanner JM. Growth at adolescence. 2a ed. Oxford: Blackwell Scientific. In: Malina RM, Bouchard C. Growth, Maturation, and physical activity. Champaign: Human Kinetics Books, 1991.

26. Matsudo SMM, and Matsudo VKR. Self-assessment and physical assessment of sexual maturation in Brazilian boys and girls: Concordance and reproducibility. Am J Hum Biol. 1994;6(4):451-5.

27. Field A. Descobrindo a estatística usando o SPSS. 2a ed. Porto Alegre: Artmed; 2009.

28. Minatto G, Petroski EL, Silva DA. Body fat, muscular and cardiorespiratory fitness according to sexual maturation among Brazilian adolescents from a town of German colonization. Rev Paul Pediatr. 2013;31(2):189-97.

29. Matton L, Duvigneaud N, Wijndaele K, Philippaerts R, Duquet W, Beunen G, et al. Secular trends in anthropometric characteristics, physical fitness, physical activity, and biological maturation in Flemish adolescents between 1969 and 2005. Am J Hum Biol. 2007;19(3):345-57.

30. Ferrari GLM, Silva LJ, Ceschini FL, Oliveira LC, Andrade DR, Matsudo VKR. Influência da maturação sexual na aptidão física em escolares do sexo masculino de Ilhabela - um estudo longitudinal. Rev Bras Ativ Fís Saúde. 2008;13(3):141-8.

31. Jones MA, Hitchen PJ, Stratton G. The importance of considering biological maturity when assessing physical fitness measures in girls and boys aged 10 to 16 years. Ann Hum Biol. 2000;27(1):57-65. 